OPEN ACCESS

Edited by:

Silvia Giordani,

Dublin City University, Ireland

Reviewed by:

Salvatore Sortino,

University of Catania, Italy

Francisco M. Raymo,

University of Miami, United States

*Correspondence:

Stefania Impellizzeri

simpellizzeri@ryerson.ca

Specialty section:

This article was submitted to

Analytical Chemistry,

a section of the journal

Frontiers in Chemistry

Received: 01 April 2020

Accepted: 05 May 2020

Published: 05 June 2020

Citation:

Trifoi LA, Hodgson GK, Dogantzis NP and Impellizzeri S (2020) A

Reconfigurable, Dual-Output INHIBIT and IMPLICATION Molecular Logic

Gate. Front. Chem. 8:470

doi: 10.3389/fchem.2020.00470

\section{A Reconfigurable, Dual-Output INHIBIT and IMPLICATION Molecular Logic Gate}

\author{
Lavinia A. Trifoi, Gregory K. Hodgson, Nicholas P. Dogantzis and Stefania Impellizzeri*
}

Laboratory for Nanomaterials and Molecular Plasmonics, Department of Chemistry and Biology, Ryerson University, Toronto, ON, Canada

Molecules that respond to input stimulations to produce detectable outputs can be exploited to mimic Boolean logic operators and reproduce basic arithmetic functions. We have designed a two-state fluorescent probe with tunable emission wavelength for the construction of a molecular logic gate with reconfigurable single- or dual-output capability. The system is based on a BODIPY skeleton coupled with 4-(dimethylamino)benzaldehyde. The behavior of the molecular logic gate can be easily investigated in solution with fluorescence spectroscopy, and the optical readout (fluorescence) can be monitored in one (green) or two (green and red) channels. Depending on the solvent of choice, single INHIBIT or dual INHIBIT/IMPLY logic functions can be achieved using chemical inputs (acid and base). Reconfiguration from single- to dual-output is thus made possible by operating the system in acetonitrile (single output) or toluene (dual output), respectively. The logic gate can be switched by manipulating the fluorescence emission via protonation or deprotonation, even when immobilized onto a glass substrate. At the solid state, the resulting output can be stored for extended periods of time. This feature provides two added benefits: (i) memory function and (ii) "set/reset" capability of the logic gate. Our design thus provides a proof-of-concept interface between the molecular and electronic domains.

Keywords: fluorescence, molecular switches, photochemistry, molecular logic gates, boron dipyrromethene, protonation

\section{INTRODUCTION}

The miniaturization race in the computer industry continues to deliver faster processors, larger capacity memory and electronic circuits with higher chip densities and computational power (International Roadmap for Devices and Systems Executive Summary, 2018). The "top-down" fabrication of smaller electronic devices and components is, however, rapidly approaching a limit due to the fact that the properties of bulk semiconductors vanish at nanometer dimensions (Vollath, 2013; Shi, 2015; Zhang, 2018; Ghatak and Mitra, 2019). Nevertheless, relentless global demand for faster data transmission and storage capacity underpins the need to investigate new materials for encoding large volumes of data at the nanoscale (Sarid and Schechtman, 2007; Reinsel et al., 2018). In computers, information processing is based on Boolean (binary) algebra. In this two-state logic framework, variables can assume only one of two values ( 1 or 0$)$ at a given time. Digital electronics require the encoding of data as electrical and optical signals in the form of such binary digits, and rely on the utilization of interconnected logic gates, i.e., idealized or physical devices implementing one or more Boolean functions (Hwang, 2016; Deschamps et al., 2017). A logic gate executes a 


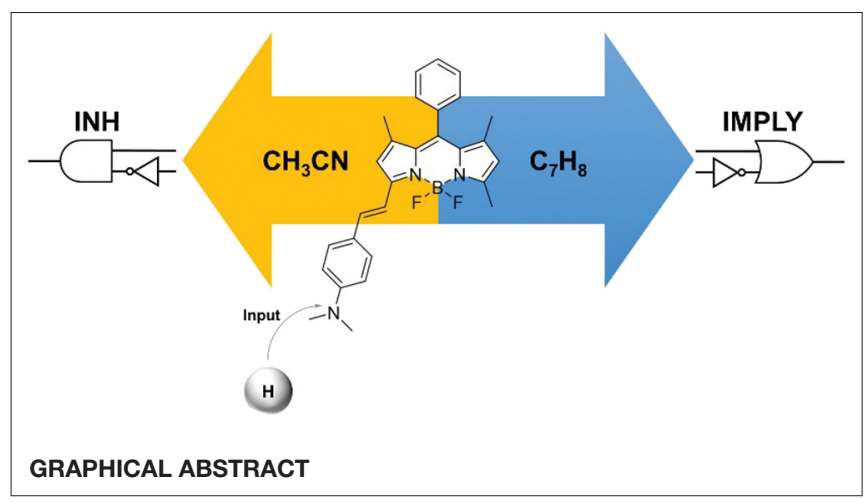

logical operation on one or more binary inputs and produces a single binary output according to convention (Gibson, 2013). Driven by the demand for higher performance capabilities, computer technology continues to stimulate the exploration of innovative materials and methodologies for the construction of logic gates. To this end, extension of information processing and computation to the molecular level can be achieved through the development of a molecular-level electronic set capable of executing functions that mimic those performed by macroscopic components (Sun et al., 2014; Mathew and Fang, 2018).

Organic molecular switches can adjust their structural, electronic and spectroscopic properties when stimulated with chemical or photonic inputs and, in return, produce a chemical, electrical and/or optical output that varies with the switching process (Feringa and Browne, 2011). The resemblance between molecular switches and logic gates is clear: they can both convert input stimulation into output signals. It follows that the principles of Boolean algebra can be applied to signal transduction operated by molecular switches. Indeed, molecular switches have long been considered valuable candidates for the realization of user-programmable electronic devices, and examples have been reported for basic (AND, NOT, and OR) and more complex (NOR, XOR, XNOR) logic functions (de Silva and Uchiyama, 2007; de Silva, 2012a,b; Szaciłowski, 2012; Stojanovic et al., 2014; Andréasson and Pischel, 2015; ErbasCakmak et al., 2018). In this context, switchable fluorescent dyes that are sensitive to external chemical stimuli provide convenient platforms for the fabrication of sophisticated molecular devices that communicate through changes in their emission properties (e.g., wavelength, quantum yield) (Daly et al., 2014). In addition, changes in emission spectra can be simultaneously observed at more than one wavelength (dual-output), resulting in the possibility of implementing more than one logic function in a single, unimolecular system (Baytekin and Akkaya, 2000; de Silva and McClenaghan, 2002; Margulies et al., 2004; Coskun et al., 2005; Shiraishi et al., 2005; Jiang and Ng, 2014; Swaminathan and Balasurbamanian, 2019).

Upon the basis of these considerations, we targeted the 3-(dimethylamino)styryl-substituted boron dipyrromethene (BODIPY) dye 1 (Scheme 1). Due to their easily accessible structural modifications and remarkable photophysical properties such as photostability, high quantum yields and

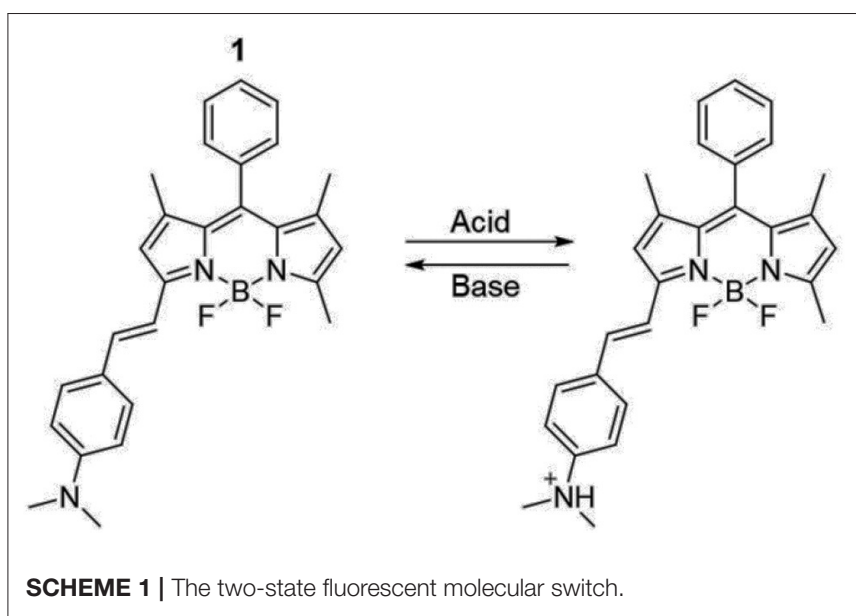

absorption coefficients, BODIPY chromophores have been successfully used for the development of selective and efficient chemosensors (Jeong and Yoon, 2012; Leen and Dehaen, 2012; Costero et al., 2018; Kaur and Singh, 2019). Extension of the BODIPY core conjugation by condensation with dialkylaminobenzaldehydes, as in $\mathbf{1}$, shifts the emission to higher wavelengths and allows the installation of a $\mathrm{pH}$-sensitive appendix that produces distinguishable changes in fluorescence upon protonation/deprotonation (Rurack et al., 2001; Baruah et al., 2006; Yu et al., 2006; Kulyk et al., 2017). Such switchable behavior has been previously exploited to construct an INHIBIT (INH) operator at the molecular level using a phenolic analog of $\mathbf{1}$ in solution (Coskun et al., 2005). Herein, we considerably expanded upon the logic capabilities of $\mathbf{1}$ by taking advantage of the solvent-dependency of its quantum yield to simultaneously achieve INH and IMPLY (logical implication) processing. The behavior of the molecular logic gate can be easily investigated in solution with fluorescence spectroscopy. Moreover, we imparted a memory function into the system by immobilizing it on a solid glass substrate, where the resulting output could be stored for extended periods of time.

\section{MATERIALS AND METHODS}

\section{Synthesis}

Syntheses were adapted from previously published protocols (Rurack et al., 2001; Coskun et al., 2005). All reaction schemes are illustrated in the Electronic Supplementary Material.

\section{Synthesis of 4,4-Difluoro-1,3,5,7-tetramethyl-8- phenyl-4-bora-3a,4a-diaza-s-indacene (0)}

In a $1000 \mathrm{~mL}$ round bottom flask, 2,4-dimethylpyrrole $(500 \mathrm{mg}$, $5.2 \mathrm{mmol}$ ), benzaldehyde ( $276 \mathrm{mg}, 2.6 \mathrm{mmol}$ ) and trifluoroacetic acid (TFA, 3 drops), were dissolved in $300 \mathrm{~mL}$ of $\mathrm{CH}_{2} \mathrm{Cl}_{2}$ and placed under $\mathrm{N}_{2}$ atmosphere. The solution was degassed for $20 \mathrm{~min}$, then stirred under nitrogen at room temperature overnight to ensure complete consumption of benzaldehyde. During the process of degassing, a color change from yellow to burnt orange to red was observed. Once TLC confirmed that benzaldehyde was consumed, a $\mathrm{CH}_{2} \mathrm{Cl}_{2}$ solution $(100 \mathrm{~mL})$ of 
DDQ (590 mg, $2.6 \mathrm{mmol}$ ) was added to the reaction mixture under air. After stirring for $20 \mathrm{~min}, 3 \mathrm{~mL}$ of $\mathrm{NEt}_{3}$ were added followed by $3 \mathrm{~mL}$ of $\mathrm{BF}_{3} \mathrm{OEt}_{2}$. After an additional $30 \mathrm{~min}$ of stirring, the reaction was extracted three times with $1: 1$ $\mathrm{H}_{2} \mathrm{O}: \mathrm{CH}_{2} \mathrm{Cl}_{2}$ and once with 1:1 brine: $\mathrm{CH}_{2} \mathrm{Cl}_{2}$. The combined organic phases were dried over $\mathrm{Na}_{2} \mathrm{SO}_{4}$ and evaporated under reduced pressure, resulting in a dark green solid. The crude compound was purified by column chromatography $\left[\mathrm{SiO}_{2}:\right.$ Hexanes/EtOAc 7:1 (v/v) $\left.+1 \% \mathrm{NEt}_{3}\right]$, affording a bright orange solid in $45.8 \%$ yield. ${ }^{1} \mathrm{H}$ NMR $\left(400 \mathrm{MHz}, \mathrm{CDCl}_{3}\right) \delta 7.50$ (d, 3H), 7.30 (d, 2H), 6.00 (s, 2H), 2.58 (s, 6H), 1.40 (s, 6H); EI-MS calculated for $\mathrm{C}_{19} \mathrm{H}_{19} \mathrm{BF}_{2} \mathrm{~N}_{2}\left(\mathrm{M}^{+}\right)$324.16; found 324.1 .

\section{Synthesis of 1}

To a clean $500 \mathrm{~mL}$ round bottom flask, compound $\mathbf{0}$ (735 mg, 0.33 $\mathrm{mmol}$ ), 4-(dimethylamino)benzaldehyde $(49.2 \mathrm{mg}, 0.33 \mathrm{mmol}$ ), toluene $(137 \mathrm{~mL})$, glacial acetic acid $(1.71 \mathrm{~mL})$ and piperidine $(2.05 \mathrm{~mL})$ were added. The solution was refluxed for $72 \mathrm{~h}$, using a Dean-Stark apparatus to remove any water formed. The crude compound was concentrated under reduced pressure and purified by column chromatography $\left[\mathrm{SiO}_{2}:\right.$ Hexanes/EtOAc 7:1 $(\mathrm{v} / \mathrm{v})]$. The blue fraction $(9.4 \%$ yield $)$ was collected and used for spectroscopic investigations. ${ }^{1} \mathrm{H}$ NMR $\left(400 \mathrm{MHz}, \mathrm{CDCl}_{3}\right) \delta 7.51-$ $7.49(\mathrm{~m}, 6 \mathrm{H}), 7.32-7.30(\mathrm{t}, 2 \mathrm{H}), 7.23-7.19(\mathrm{~d}, 2 \mathrm{H}), 6.67-6.72(\mathrm{~m}$, $2 \mathrm{H}), 6.59(\mathrm{~s}, 1 \mathrm{H}), 5.96(\mathrm{~s}, 1 \mathrm{H}), 3.03-3.04(\mathrm{~d}, 6 \mathrm{H}), 2.59(\mathrm{~s}, 3 \mathrm{H})$, $1.42(\mathrm{~s}, 3 \mathrm{H}), 1.38$ (s, 3H).

\section{Synthesis of 4,4-Difluoro-8(4'-hydroxyphenyl)- 1,3,5,7-tetramethyl-4-bora-3a,4a-diaza-s-inda-cene (Ob)}

To a clean $1000 \mathrm{~mL}$ round bottom flask, 2,4-dimethylpyrrole (500 mg, $5.26 \mathrm{mmol}$ ), 4-hydroxybenzaldehyde (320 mg, 2.6 mmol), TFA ( 3 drops) and $\mathrm{CH}_{2} \mathrm{Cl}_{2}(300 \mathrm{~mL})$ were added. The reaction was placed under $\mathrm{N}_{2}$ atmosphere and degassed for $20 \mathrm{~min}$. A color change from yellow to orange to deep red was observed. The solution was stirred at room temperature under inert conditions for $24 \mathrm{~h}$. Once the consumption of 4 hydroxybenzaldehyde was confirmed by TLC, DDQ (590 mg, $2.6 \mathrm{mmol}$ ) dissolved in $100 \mathrm{~mL}$ of $\mathrm{CH}_{2} \mathrm{Cl}_{2}$ was added to the reaction mixture under air. The solution was stirred for $30 \mathrm{~min}$ followed by addition of $\mathrm{NEt}_{3}(3 \mathrm{~mL})$ and then $\mathrm{BF}_{3} \mathrm{OEt}_{2}(3 \mathrm{~mL})$. After $30 \mathrm{~min}$ of stirring, the solution was washed three times with 1:1 $\mathrm{H}_{2} \mathrm{O}: \mathrm{CH}_{2} \mathrm{Cl}_{2}$ and once with 1:1 brine: $\mathrm{CH}_{2} \mathrm{Cl}_{2}$. The combined organic layers were dried over $\mathrm{Na}_{2} \mathrm{SO}_{4}$, evaporated under reduced pressure and used for the next step without further purification. Yield 38.5\%. ${ }^{1} \mathrm{H}$ NMR $\left(400 \mathrm{MHz}, \mathrm{CDCl}_{3}\right) \delta 7.53(\mathrm{~d}$, $2 \mathrm{H}), 6.95(\mathrm{~d}, 2 \mathrm{H}), 6.59(\mathrm{~s}, 3 \mathrm{H}), 3.02-3.09(\mathrm{t}, 6 \mathrm{H}), 2.48(\mathrm{~s}, 3 \mathrm{H}), 1.45$ (s, 3H), $1.42(\mathrm{~d}, 2 \mathrm{H})$.

\section{Synthesis of 2}

In a clean, dry $1000 \mathrm{~mL}$ flask, compound $\mathbf{0 b}(310 \mathrm{mg}, 0.91 \mathrm{mmol})$ and 4-(dimethylamino)benzaldehyde ( $136 \mathrm{mg}, 0.91 \mathrm{mmol}$ ) were dissolved in toluene $(65 \mathrm{~mL})$, followed by the addition of glacial acetic acid $(0.7 \mathrm{~mL})$, and piperidine $(0.9 \mathrm{~mL})$. The solution was refluxed for $72 \mathrm{~h}$, using a Dean-Stark apparatus to remove any water formed during the reaction. The crude compound was concentrated under reduced pressure and purified by column chromatography $\left[\mathrm{SiO}_{2}\right.$ : Hexanes/EtOAc 1:1 (v/v) then $\mathrm{CH}_{2} \mathrm{Cl}_{2} / \mathrm{MeOH}$ 95:1 (v/v)] The blue fraction was collected and used immediately for the next step ( $4.2 \%$ yield). FTIR-ATR: 3330 $\mathrm{cm}^{-1}\left(\nu_{\mathrm{s}}, \mathrm{O}-\mathrm{H}\right) ; 3000,2930,2885 \mathrm{~cm}^{-1}\left(\nu_{\mathrm{s}}, \mathrm{C}-\mathrm{H}\right) ; 1636 \mathrm{~cm}^{-1}$ $\left(\nu_{\mathrm{s}}, \mathrm{C}=\mathrm{C}\right.$, alkene and aromatic); $1560 \mathrm{~cm}^{-1}\left(\nu_{\mathrm{b}}, \mathrm{N}-\mathrm{H}\right) ; 1447$ $\mathrm{cm}^{-1}\left(\nu_{\mathrm{s}}\right.$, aromatic $\left.\mathrm{C}=\mathrm{C}\right) ; 1390 \mathrm{~cm}^{-1}\left(\nu_{\mathrm{b}}, \mathrm{CH}_{3}\right) ; 1265 \mathrm{~cm}^{-1}\left(\nu_{\mathrm{s}}\right.$, C-N aromatic); $1060 \mathrm{~cm}^{-1}\left(\nu_{\mathrm{s}}, \mathrm{C}-\mathrm{O}\right) ; 947 \mathrm{~cm}^{-1}\left(\nu_{\mathrm{b}}, \mathrm{O}-\mathrm{H}\right) ; 766$ $\mathrm{cm}^{-1}\left(\nu_{\mathrm{b}}, \mathrm{C}-\mathrm{H}\right.$, oop$)$.

\section{Synthesis of 3}

In a clean $100 \mathrm{~mL}$ two-neck round bottom flask, a stir bar and compound $2(3.5 \mathrm{mg}, 7.4 \mu \mathrm{mol})$ were added. The system was placed under $\mathrm{N}_{2}$ atmosphere and allowed to degas for $30 \mathrm{~min}$ followed by the addition of dry THF $(18.7 \mathrm{~mL})$. Maintaining inert conditions, 3-(triethoxysilyl)propyl isocyanate $(37.4 \mu \mathrm{L}$, $151 \mu \mathrm{mol})$ was added and the reaction was refluxed at $70^{\circ} \mathrm{C}$ for $24 \mathrm{~h}$. The reaction mixture was then concentrated under reduced pressure (mass obtained: $13.7 \mathrm{mg}$ ) and used for functionalization of glass slides without further purification. FTIR-ATR: 3000, $2910,2870 \mathrm{~cm}^{-1}\left(\nu_{s}, \mathrm{C}-\mathrm{H}\right) ; 1720 \mathrm{~cm}^{-1}\left(\nu_{\mathrm{s}}, \mathrm{C}=\mathrm{O}\right) ; 1600 \mathrm{~cm}^{-1}$ $\left(\nu_{\mathrm{s}}, \mathrm{C}=\mathrm{C}\right.$, alkene and aromatic $) ; 1515 \mathrm{~cm}^{-1}\left(\nu_{\mathrm{b}}, \mathrm{N}-\mathrm{H}\right) ; 1456$ $\mathrm{cm}^{-1}\left(\nu_{\mathrm{s}}\right.$, aromatic $\left.\mathrm{C}=\mathrm{C}\right) ; 1380 \mathrm{~cm}^{-1}\left(\nu_{\mathrm{b}}, \mathrm{CH}_{3}\right) ; 1264 \mathrm{~cm}^{-1}\left(\nu_{\mathrm{s}}\right.$, C-N aromatic); $1113 \mathrm{~cm}^{-1}\left(\nu_{\mathrm{s}}, \mathrm{Si}-\mathrm{CH}_{2}\right) ; 1065 \mathrm{~cm}^{-1}\left(\nu_{\mathrm{s}}, \mathrm{Si}-\mathrm{OEt}\right)$; $1040 \mathrm{~cm}^{-1}\left(\nu_{\mathrm{s}}, \mathrm{C}-\mathrm{O}\right) ; 806,740 \mathrm{~cm}^{-1}\left(\nu_{\mathrm{b}}, \mathrm{C}-\mathrm{H}\right.$, oop$)$.

\section{Methods}

2,4-dimethylpyrrole, benzaldehyde, 2,3-dichloro-5,6-dicyano1,4-benzoquinone (DDQ) and boron trifluoride diethyl etherate $\left(\mathrm{BF}_{3} \mathrm{OEt}_{2}\right)$ were purchased from Sigma Aldrich. Solvents and trifluoroacetic acid (TFA) were purchased from ACP. Dry $\mathrm{CH}_{2} \mathrm{Cl}_{2}$ and THF were purified with an MBraun MBM-SPS solvent purification system. Ultrapure deionized water (Milli $\Omega$, 18.2 M $\Omega$ ) was obtained from a Millipore Purification System. Reactions were monitored by thin layer chromatography using aluminum backed sheets coated with $200 \mu \mathrm{m}$ silica $\left(60, \mathrm{~F}_{254}\right)$. SiliaFlash ${ }^{\circledR}$ P60, 40-63 mm (230-400 mesh) silica gel from SiliCycle was used for purification of compounds by column chromatography. Mass spectral analysis was performed with a 7890B GC System equipped with a 5977 mass selective detector from Agilent Technologies. NMR spectra were recorded at room temperature with a Bruker Avance 400 spectrometer. Steadystate absorption spectra were recorded with an Agilent Cary 60 UV-visible spectrometer, using quartz cells with a path length of $1 \mathrm{~cm}$. Steady-state emission spectra of aerated solutions as well as functionalized and unfunctionalized glass slides were recorded with an Agilent Cary Eclipse spectrometer operating in right-angle geometry. Diffuse reflectance measurements were performed using an Agilent Cary 5000 spectrophotometer equipped with a DRA-2500 accessory. FTIR Diamond ATR spectra were recorded with a Cary 630 spectrometer by Agilent Technologies. Glass slides were soaked in piranha solution (3:1 $\mathrm{H}_{2} \mathrm{SO}_{4}: \mathrm{H}_{2} \mathrm{O}_{2}$ ) for $1 \mathrm{~h}$ followed by several rinses with deionized and Milli $\Omega$ water, respectively. The slides were then stored in Milli $\Omega$ water for future use. Crude compound $3(13.7 \mathrm{mg})$ was then dissolved in $1 \mathrm{~mL}$ of toluene and $300 \mu \mathrm{L}$ of this solution was drop casted onto each slide. Wet slides were allowed to dry inside a fume hood for 5 days until all the solvent evaporated. 


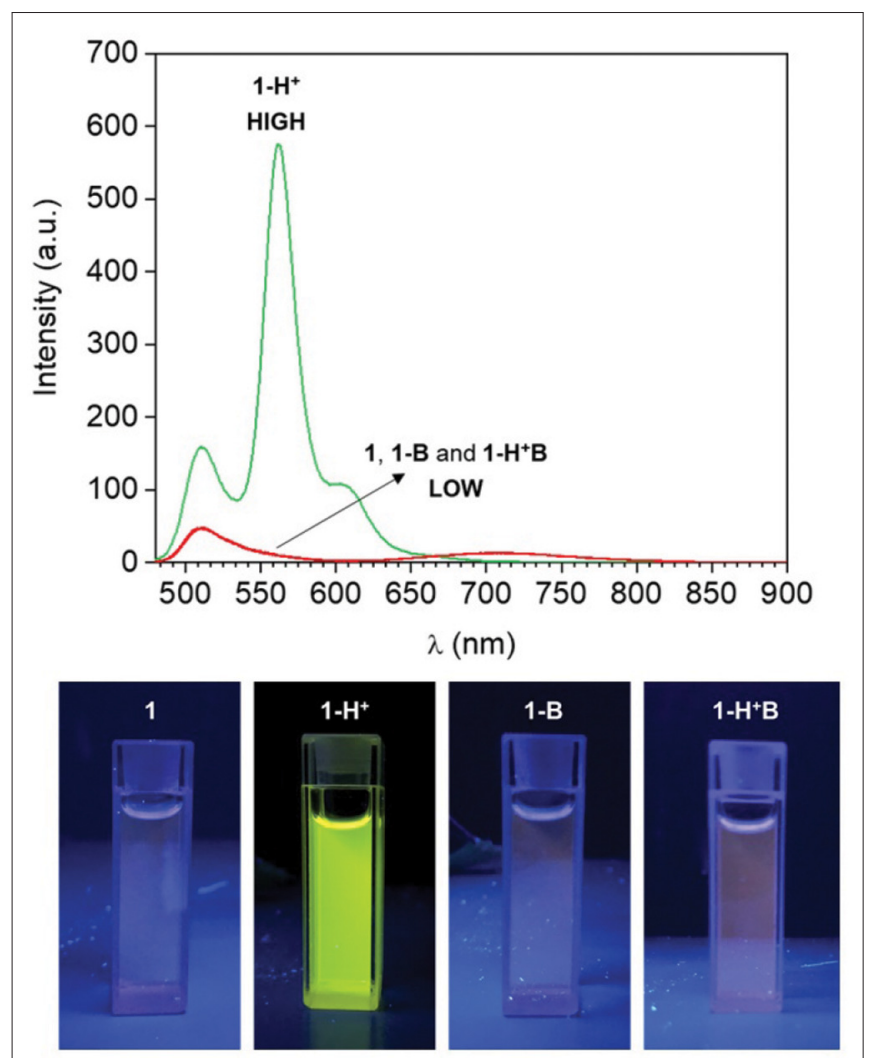

FIGURE 1 | (Top) Emission spectra of $\mathrm{C} \mathrm{CH}_{3} \mathrm{CN}$ solution of $\mathbf{1}\left(1 \mu \mathrm{M}, 20^{\circ} \mathrm{C}\right.$, $\lambda_{\mathrm{Ex}}=460 \mathrm{~nm}$ ) before (1) and after the addition of 50 eq. $\mathrm{HClO}_{4}\left(\mathbf{1}-\mathbf{H}^{+}\right), 50$ eq. $\mathrm{NEt}_{3}$ (1-B) and 50 eq. of $\mathrm{HClO}_{4}+50$ eq. $\mathrm{NEt}_{3}\left(\mathbf{1}-\mathbf{H}^{+} \mathbf{B}\right)$. (Bottom) Digital photographs of the same solutions illuminated at $365 \mathrm{~nm}$ with a laboratory UV lamp.

Functionalized slides were thoroughly rinsed with toluene and subjected to one full acid/base cycle for equilibration.

\section{RESULTS AND DISCUSSION}

\section{Solution Spectroscopy (Combinatorial Logic)}

Compound $\mathbf{1}$ is based on a BODIPY fluorophore, where a 4-(dimethylamino)styryl group was used to replace one of the pyrrolic methyls. This transformation extends the conjugation of the adjacent BODIPY core, shifting its absorption band bathochromically to $\lambda_{\mathrm{Abs}}=598 \mathrm{~nm}$ (Figure S1, Electronic Supplementary Material). Spectroscopic data from our (Figure 1) and other (Rurack et al., 2001; Baruah et al., 2006; Yu et al., 2006) laboratories have shown that a broad emission band for 1 in $\mathrm{CH}_{3} \mathrm{CN}$ appears at $\lambda_{\mathrm{Em}}=710 \mathrm{~nm}$ due to an intramolecular charge transfer (ICT) process between the tertiary amino group, which possesses strong electron donating properties, and the electron-deficient boron-dipyrromethene. In addition, the fluorescence of $\mathbf{1}$ is almost completely quenched in $\mathrm{CH}_{3} \mathrm{CN}$ ( 1 in Figure 1) due to accelerated internal conversion (Baruah et al., 2006). Protonation of the tertiary amine upon addition of 50 equivalents of $\mathrm{HClO}_{4}$ to $\mathbf{1}$ dramatically alters
TABLE 1 | Truth table of the two-state molecular switch in $\mathrm{CH}_{3} \mathrm{CN}^{a-c}$.

\begin{tabular}{lccc}
\hline & \multicolumn{1}{c}{ Input data } & & Output data \\
IN1 & IN2 & O \\
\hline 0 & 0 & 0 \\
0 & 1 & 0 \\
1 & 0 & 1 \\
1 & 1 & 0 \\
\hline
\end{tabular}

a The switching process is illustrated in Scheme 1.

${ }^{b}$ The two inputs IN1 and IN2 are acid $\left(\mathrm{H}^{+}\right)$and base (B). The input strings where IN1 and IN2 are both 1 imply that acid and base are both present.

${ }^{c}$ The output $O$ is the fluorescence at $565 \mathrm{~nm}$. A positive logic convention (off $=0$, on $=$ 1) was applied to encode the binary digits for the two inputs and the output.

its electron donating properties and consequently suppresses the charge transfer interaction. As a result, both absorption (Figure S1) and emission (Figure 1) spectra of $\mathbf{1 - \mathbf { H } ^ { + }}$ shift toward shorter wavelengths $\left(\lambda_{\mathrm{Abs}}=550 \mathrm{~nm}\right.$ and $\left.\lambda_{\mathrm{Em}}=565 \mathrm{~nm}\right)$, mimicking the typical BODIPY-like narrow, structured bands. In contrast to its unprotonated form, $\mathbf{1 - \mathbf { H } ^ { + }}$ is highly fluorescent. Reversible deprotonation of $\mathbf{1}-\mathbf{H}^{+}$to $\mathbf{1}$ upon the addition of 50 equivalents of triethylamine $\left(\mathrm{NEt}_{3}\right)$ restores the original bands $\left(\mathbf{1 - H ^ { + }} \boldsymbol{B}\right.$ in Figure 1, Figure S1), while prior addition of $\mathrm{NEt}_{3}$ to the unprotonated dye (1-B in Figure $\mathbf{1}$ and Figure S1) has no effect on its spectroscopic properties. This behavior is also illustrated by digital photographs of the same solutions illuminated at $365 \mathrm{~nm}$ with a laboratory UV lamp (Figure 1).

$\mathrm{HClO}_{4}$ (Input 1, IN1) and $\mathrm{NEt}_{3}$ (Input 2, IN2) can be either absent (OFF) or present (ON). Binary digits can thus be encoded such that the acid and base inputs can be logically processed using positive logic convention ( $\mathrm{OFF}=0$ and $\mathrm{ON}=1$ ). Under our excitation conditions, the fluorescence intensity of $\mathbf{1}$ at $565 \mathrm{~nm}$ (Output, O) was measured at approximately 10 a.u. in the unprotonated form, but grows to 550 a.u. in the presence of acid (Figure 1). Assigning a threshold of 200 a.u. $(I<200$ a.u. $=$ LOW and $I \geq 200$ a.u. $=\mathrm{HIGH}$ ) and once again applying positive logic conventions ( $\mathrm{LOW}=0, \mathrm{HIGH}=1$ ), this chemical system responds to an input string of two binary digits (IN1 and IN2) producing a binary output $(\mathrm{O})$. For example, the input string $(0$, 0 ) indicates that both input stimuli, acid and base, are absent. Under these conditions, $\mathbf{1}$ is in its unprotonated form and the emission in the green channel (at $565 \mathrm{~nm}$ ) is below the ONOFF threshold; the output is 0 . In contrast, an input string $(1,0)$ indicates that the acid input is present. Under these conditions, the dominant species is $\mathbf{1 - \mathbf { H } ^ { + }}$ and the fluorescence at $565 \mathrm{~nm}$ is high; the output is 1 . Following this convention, the four output strings corresponding to the four possible combinations of input strings can be determined (Table 1 and Figure 2). From Table 1, it can be observed that the logic behavior of the twostate molecular switch in $\mathrm{CH}_{3} \mathrm{CN}$ corresponds to that of a combinatorial INH logic circuit (Figure 2).

Interestingly, when the molecular switch $\mathbf{1}$ is operated in $\mathrm{CH}_{3} \mathrm{CN}$, the fluorescence in the red wavelength channel is excluded by the molecular processor, due to the low quantum

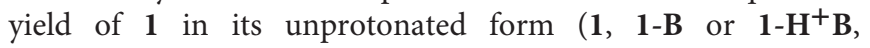
Figure 1). Nevertheless, the nonradiative quenching constant 


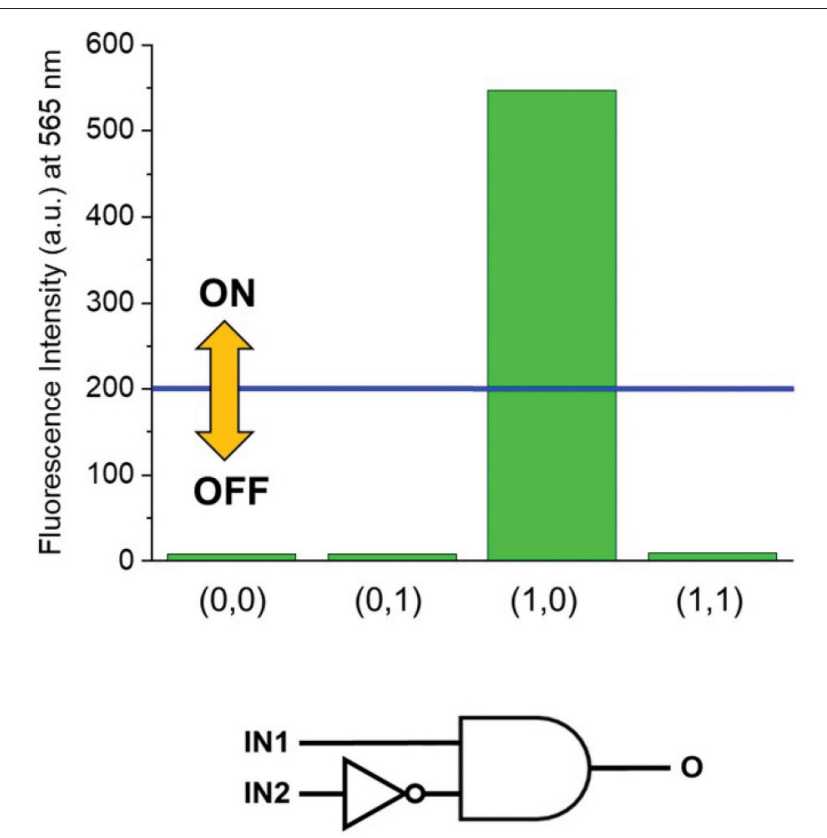

FIGURE 2 | (Top) Column diagram of the fluorescence intensities at $565 \mathrm{~nm}$. The blue line shows the ON-OFF threshold. (Bottom) The INH logic circuit for the two-state molecular switch when operated in $\mathrm{CH}_{3} \mathrm{CN}$.

$k_{\mathrm{nr}}$ reduces from $7.58 \times 10^{8} \mathrm{~s}^{-1}$ in $\mathrm{CH}_{3} \mathrm{CN}$ to $0.46 \times 10^{8}$ $\mathrm{s}^{-1}$ in $\mathrm{C}_{7} \mathrm{H}_{8}$ (Baruah et al., 2006). In agreement with these data, as well as other examples of polarity-sensitive dyes for which emission is substantially increased with a decrease in solvent polarity (Wagner et al., 2018), the emission spectrum of compound $\mathbf{1}$ dissolved in toluene ( 1 in Figure 3 ) displays a strong fluorescence band. Noticeably, the emission maximum shifts hypsochromically from $710 \mathrm{~nm}$ in $\mathrm{CH}_{3} \mathrm{CN}$ (polar solvent) to $640 \mathrm{~nm}$ in $\mathrm{C}_{7} \mathrm{H}_{8}$ (non-polar solvent) and is accompanied by a gain of fine structure and a decrease in bandwidth. This is a well-known phenomenon for (dimethylamino)styryl boron dipyrromethene dyes. Quantum mechanical calculations (Baruah et al., 2006) have confirmed that an ICT process is responsible for the observed solvent sensitivity, with polar aprotic solvents such as $\mathrm{CH}_{3} \mathrm{CN}$ stabilizing the excited state and thus red-shifting the emission. Similarly to $\mathrm{CH}_{3} \mathrm{CN}$, subjecting 1 to protonation by addition of 50 equivalents of $\mathrm{HClO}_{4}$ in $\mathrm{C}_{7} \mathrm{H}_{8}$ switches the absorption (Figure S2) and emission $\left(\mathbf{1}-\mathrm{H}^{+}\right.$in Figure 3) to lower wavelengths, as evidenced by the appearance of a strong fluorescence band at $565 \mathrm{~nm}$ and almost complete disappearance of the red fluorescence at $640 \mathrm{~nm}$. Similar to the behavior observed in acetonitrile, addition of $\mathrm{NEt}_{3}$ to the unprotonated dye (1-B in Figure 3, Figure S2) has no remarkable effect on its spectroscopic properties, while deprotonation of $\mathbf{1 - \mathbf { H } ^ { + }}$ to $\mathbf{1}$ reinstates the original bands $\left(\mathbf{1 - H ^ { + }} \boldsymbol{B}\right.$ in Figure 3, Figure S2).

As visibly shown by the digital photographs reported in Figure 3, the dual fluorescence of $\mathbf{1}$ in $\mathrm{C}_{7} \mathrm{H}_{8}$ translates into the possibility of simultaneously monitoring two separate outputs. Indeed, a change in emission above the set threshold can now be recorded at two different channels, $\lambda_{\mathrm{Em}}=565 \mathrm{~nm}$ (Output

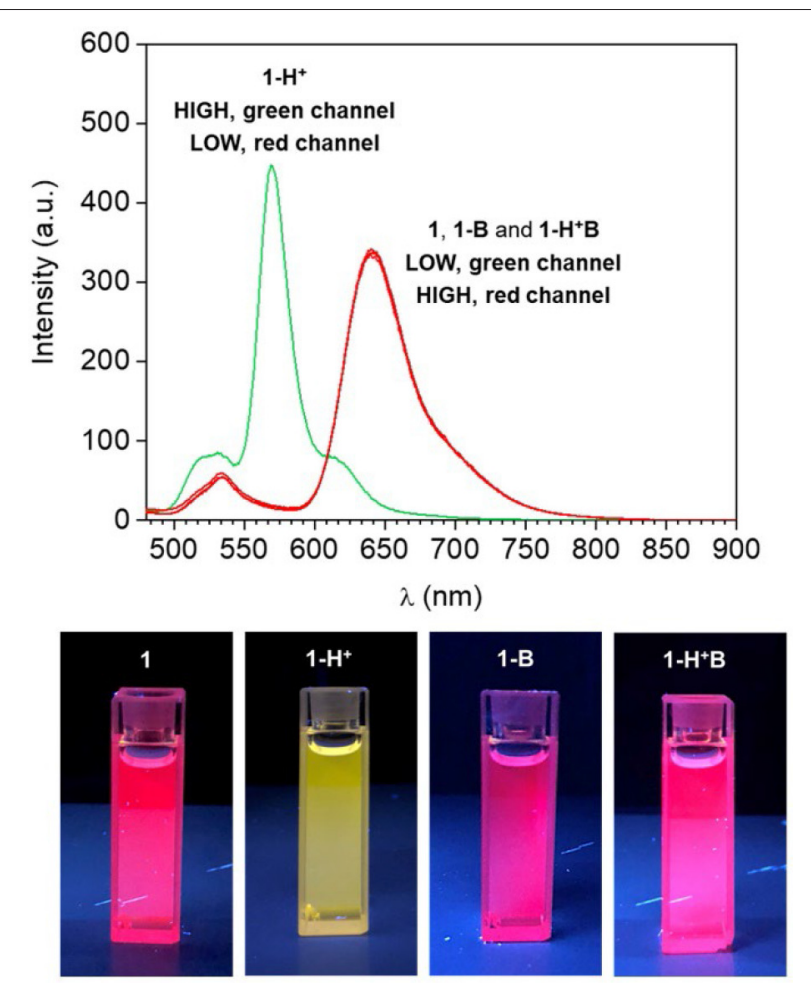

FIGURE 3 | (Top) Emission spectra of a $\mathrm{C}_{7} \mathrm{H}_{8}$ solution of $\mathbf{1}$ $\left(1 \mu \mathrm{M}, 20^{\circ} \mathrm{C}, \lambda_{\mathrm{Ex}}=460 \mathrm{~nm}\right)$ before (1) and after the addition of $50 \mathrm{eq} . \mathrm{HClO}_{4}$ $\left(\mathbf{1}-\mathbf{H}^{+}\right), 50$ eq. $\mathrm{NEt}_{3}(\mathbf{1}-\mathbf{B})$ and 50 eq. of $\mathrm{HClO}_{4}+50$ eq. NEt $3\left(\mathbf{1}-\mathbf{H}^{+} \mathbf{B}\right)$. (Bottom) Digital photographs of the same solutions illuminated at $365 \mathrm{~nm}$ with a laboratory UV lamp.

1) or $\lambda_{\mathrm{Em}}=640 \mathrm{~nm}$ (Output 2). Using the same threshold value of 200 a.u. $(I<200$ a.u. $=$ LOW and $I \geq 200$ a.u. $=\mathrm{HIGH})$ and applying positive logic convention (LOW $=0, \mathrm{HIGH}=$ 1 ), this chemical system responds to an input string of two binary digits (IN1 and IN2) producing two binary outputs (O1 and $\mathrm{O} 2$ ) in toluene. The four output strings corresponding to the four possible combinations of input strings can be determined for the two outputs independently (Table 2). The results presented in Table 2 illustrate that the logic behavior of the two-state molecular switch in $\mathrm{C}_{7} \mathrm{H}_{8}$ corresponds to that of a combinatorial INH logic circuit when the output is monitored in the green channel (O1), or as an IMPLY (Figure 4) circuit when tracked in the red channel (O2). The molecular operator can thus be instantaneously reconfigured simply by the selection of monitoring wavelength.

\section{Solid State Spectroscopy (Sequential Logic)}

In digital circuit theory, combinational logic refers to circuits whose outputs are a function of the present value of the inputs only (Lipiansky, 2012). In other words, the outputs obtained by the logic operator are purely a function of the inputs. If the inputs are changed, the outputs are changed (time independent logic). It follows that in combinatorial systems, any information about the previous status of the inputs is lost. Combinational logic 
TABLE 2 | Truth table of the two-state molecular switch in $\mathrm{C}_{7} \mathrm{H}_{8}{ }^{a-c}$.

\begin{tabular}{lcccc}
\hline & Input data & & \multicolumn{2}{c}{ Output data } \\
\cline { 3 - 4 } IN1 & IN2 & & O1 & O2 \\
\hline 0 & 0 & 0 & 1 \\
0 & 1 & 0 & 1 \\
1 & 0 & 1 & 0 \\
1 & 1 & 0 & 1 \\
\hline
\end{tabular}

a The switching process is illustrated in Scheme 1.

${ }^{b}$ The two inputs IN1 and IN2 are acid $\left(\mathrm{H}^{+}\right)$and base $(\mathrm{B})$. The input strings where IN1 and IN2 are both $1 \mathrm{imply}$ that acid and base are both present.

${ }^{c}$ Output 01 is fluorescence at $565 \mathrm{~nm}$, while output $\mathrm{O} 2$ is fluorescence at $640 \mathrm{~nm}$. A positive logic convention (off $=0$, on $=1$ ) was applied to encode the binary digits for the inputs and outputs.

circuits have no memory function because the current output state cannot be stored, and thus has no consequence for the next resulting output state. No feedback is required; the operator's output simply reflects the current status of the inputs according to the logic convention obeyed by the logic gate. Consequently, such systems cannot be used to store data. Upon the basis of these considerations, the behavior of the molecular switch $\mathbf{1}$ in solution can be classified as combinatorial logic. Even though the choice of solvent system $\left(\mathrm{CH}_{3} \mathrm{CN}\right.$ vs. $\left.\mathrm{C}_{7} \mathrm{H}_{8}\right)$ determines the particular arithmetic capabilities of the logic gate, its behavior lacks memory regardless. In contrast, the output of sequential logic circuits depends not only upon the latest inputs, but also on the condition of earlier inputs. In sequential logic, the output is a function of the present inputs as well as the previous states of the system, and involves feedback from output to input that is stored in the system's memory for the next operation (Lipiansky, 2012). Such systems can be used to store data for extended periods of time. To implement sequential character into the molecular logic gate $\mathbf{1}$, its chemical structure was synthetically modified by insertion of a hydroxyl group (2, ESI) followed by reaction with 3-(triethoxysilyl)propyl isocyanate (ICPTES) to yield compound 3. Successful modification of $\mathbf{2}$ with the silane cap was confirmed by FTIR-ATR spectroscopy (Figure S3). Specifically, the FTIRATR spectrum of 3 shows the appearance of a peak at $\sim 1720$ $\mathrm{cm}^{-1}$ attributable to the $\mathrm{C}=\mathrm{O}$ stretching vibration and the sharp, well-defined doublet for the stretching absorptions of $\mathrm{Si}-\mathrm{CH}_{2}$ at 1065 and $\mathrm{Si}-\mathrm{OEt}$ at $1110 \mathrm{~cm}^{-1}$. Furthermore, the $\mathrm{OH}$ stretching absorption at $3330 \mathrm{~cm}^{-1}$, clearly identified in the spectrum of 2, disappears in that of the capped molecule. Without further purification, compound 3 was dissolved in $\mathrm{C}_{7} \mathrm{H}_{8}$ and drop casted onto a clean glass slide (Figure 5). The slides were subsequently washed with $\mathrm{C}_{7} \mathrm{H}_{8}$ to remove any unbound 3 or trace amounts of residual 2, then dried prior to use. The presence of 3 was confirmed by diffuse reflectance spectroscopy (Figure S4). Note that Figure 5 illustrates the mode of chemical attachment of 3 to the glass support, but should not be interpreted as conclusive evidence of the formation of a uniform or nonuniform monolayer. Nevertheless, the number of dye molecules immobilized on the glass support was estimated to be on the order of $10^{13} \mathrm{~cm}^{-2}$, which is within the expected range for a non-uniform monolayer (ESI).

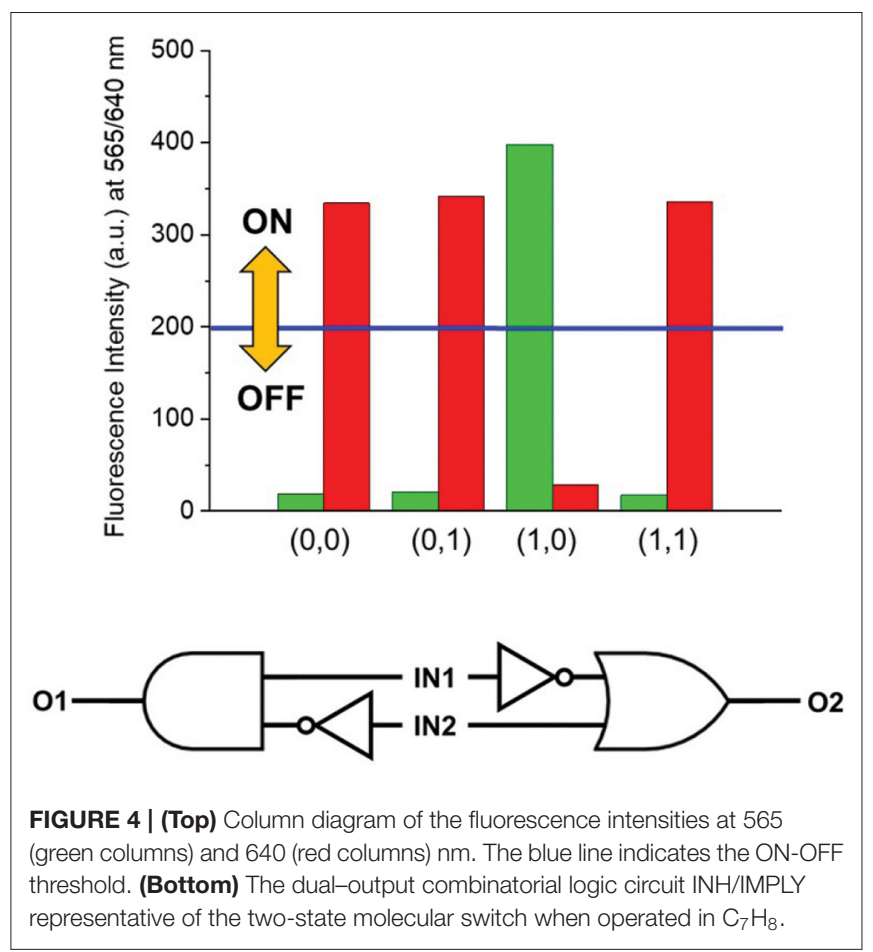

With our design, protonation/deprotonation of the molecular switch allows for effective fluorescence modulation at the solid state. Specifically, the fluorescence of $\mathbf{3}$ is partially quenched (3 in Figure S5) as a result of the formation of aggregated clusters, which favors non-radiative decay processes from the excited state due to $\pi-\pi$ stacking. After functionalized glass slides were immersed in $15 \mathrm{mM} \mathrm{HClO}_{4}$ in ethanol for $30 \mathrm{~min}$, and subsequently washed with $\mathrm{EtOH}$ and $\mathrm{Milli} \Omega$ water, the emission intensity increased substantially $\left(\mathbf{3}-\boldsymbol{H}^{+}\right.$in Figure S5). We also observed a $45 \mathrm{~nm}$ hypsochromic shift, with $\lambda_{\text {Em }}$ shifting from $565 \mathrm{~nm}$ in solution to $520 \mathrm{~nm}$ at the solid state (compare Figures 1, 3 with Figure S5). Both the increase in intensity and blue-shift of the fluorescence upon protonation are consistent with a disruption of $\pi-\pi$ stacking interactions by augmented Coulombic repulsion and possible geometric rearrangement into a twisted conformation in the solid state. Such a configuration would also lead to less effective delocalization, which is likely responsible for the observed blue shift. It has also been suggested that blue-shifted emission at the solid state can occur as a result of lower reorganization energy in aggregates compared to solution (Wu et al., 2014). Moreover, the film can be dipped into a $40 \mathrm{mM}$ ethanolic solution of $\mathrm{NaOH}$ for $30 \mathrm{~min}$ to deprotonate 3$\mathbf{H}^{+}$and regenerate 3 , concomitantly lowering the fluorescence permanently, or until the system is once again subjected to an acid input (Figure 6). No fluorescence emission was observed for an unfunctionalized glass slide, before or after treatment with acid or base (Figure S6). Thus, the covalent attachment of 3 to a glass substrate permits long-term storage of the resulting emission readout (output) even after the removal of the system from acid or base inputs. This practical benefit generates a feedback loop by allowing the current state of 


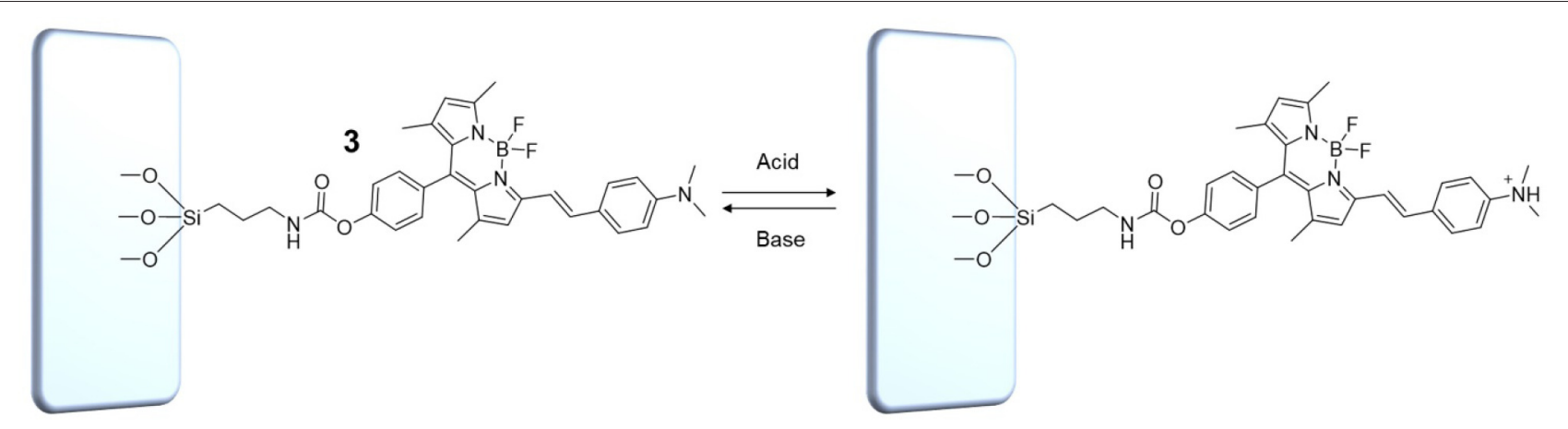

FIGURE 5 | Protonated and unprotonated forms of the molecular switch $\mathbf{3}$ immobilized on a solid glass support.

the molecular switch to influence the next output state upon interaction with new inputs. As such, we can transduce the acidification of $\mathbf{3}$ into a "set" operation, with fluorescence at $520 \mathrm{~nm}$ defined as the output $(\mathrm{O})$. Under this condition, the addition of IN1 (acid) can steadily "set" ( $S$ or write) the state of the molecular switch to 1 , and addition of IN2 (base) would "reset" ( $R$ or erase) the system to state 0 . Figure 6 illustrates the $S-R$ mode transduced by the bistable solid state switch over several acid/base cycles, operating according to the INH convention set. Noticeably, the switching behavior at the solid state is representative of a single-output logic system, where only the fluorescence in the green channel can be modulated by the acid and base input combinations. In fact, no fluorescence is observed at higher wavelengths (Figure S5). Such behavior mirrors what is observed for the molecular switch $\mathbf{1}$ dissolved in a polar solvent $\left(\mathrm{CH}_{3} \mathrm{CN}\right)$, which is known to accelerate internal conversion and consequently quenches the fluorescence of the unprotonated species. It is possible that residual hydroxyl groups remaining on the glass surface after treatment with piranha solution are responsible for the relatively high polarity of the local environment, which commonly decreases the emission intensity, and hypsochromically shifts the absorption (compare Figures S1, S2, S4; Wagner et al., 2018). The utilization of a polar protic solvent $(\mathrm{EtOH})$ for the immersion solutions would also have maintained a polar environment during the switching cycles. This rationale is also supported by the fact that a methoxy analog of compound 1 (Baruah et al., 2006) was noted to be almost completely quenched in $\mathrm{MeOH}$.

\section{CONCLUSIONS}

The growing demand for smaller and faster digital devices continues to stimulate the exploration of innovative materials for information processing. Molecules that respond to changes in their environment can simulate the behavior of the fundamental components of integrated circuits. Particularly, fluorescent chemosensors are able to switch from a non-emissive state to a fluorescent one, or change the color of emission in response to chemical inputs. The mechanisms responsible for fluorescence switching require coupling a selective chemical reaction to a photophysical process (emission). The observable changes in the

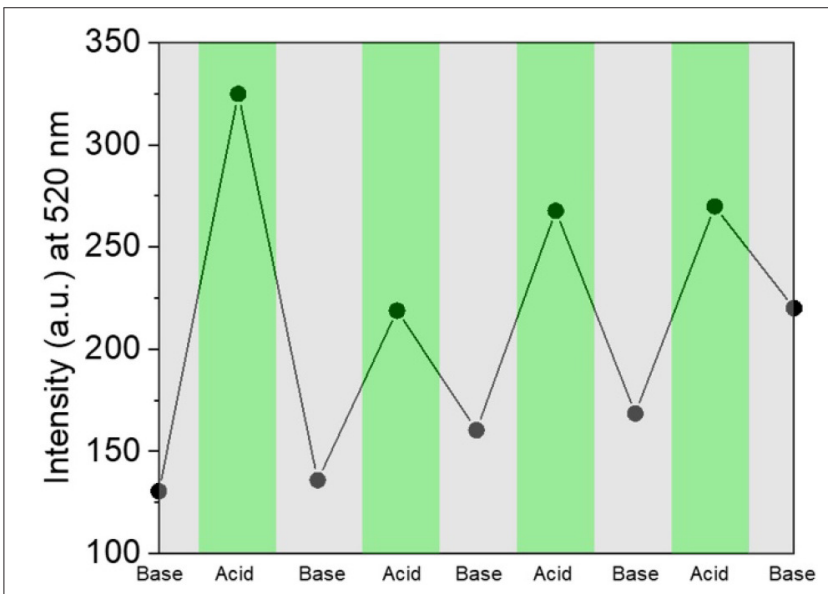

FIGURE 6 | Fluorescence intensity recorded at $520 \mathrm{~nm}$ upon several protonation/deprotonation cycles performed upon $\mathbf{3}$ immobilized on a glass slide.

emission of these probes are the result of the complex interplay between excited state dynamics and environmental parameters.

Herein, a bistable unimolecular system based upon the covalent conjugation between a boron-dipyrromethene chromophore and 4-(dimethylamino)benzaldehyde was synthesized in two steps and its environmentally responsive optical characteristics were investigated by steady-state absorption and emission spectroscopy. The system displays solvent and $\mathrm{pH}$ dependent emission in solution: the fluorescence quantum yield decreases in a polar aprotic solvent $\left(\mathrm{CH}_{3} \mathrm{CN}\right)$ and experiences a large bathochromic shift compared to the molecule dissolved in non-polar aprotic solvent $\left(\mathrm{C}_{7} \mathrm{H}_{8}\right)$. In both solvents, large spectral shifts are observed in both absorption and emission spectra upon protonation of the tertiary amine. Interconversion between the protonated and neutral forms can be induced by subjecting the system to an acid or base input, and can be monitored by measuring the fluorescence of the two forms. Interplay between the two input stimuli can be exploited to transduce logic operations at the molecular level. Remarkably, the solvent dependency of the emission allows for the implementation of two different logic operators within 
the same system. Specifically, the logic function executed by this molecular switch in $\mathrm{CH}_{3} \mathrm{CN}$ is equivalent to that of a combinatorial single-output circuit incorporating an INHIBIT gate; in contrast, a combinatorial dual-output INHIBIT/IMPLY gate can be accessed by dissolving the system in $\mathrm{C}_{7} \mathrm{H}_{8}$. In both cases, positive logic conventions can be used to encode binary digits into the chemical inputs and optical outputs. Thus, the molecular system can be easily reconfigured between two modes (logic functions) simply by changing the operating medium. Surface immobilization of the molecular switch onto a solid support instilled sequential (programmable) character and memory, where the resulting output data can be stored for extended periods of time even after removal of the inputs, demonstrating the potential for integration into solid state technology. While the goal of this work was to study the viability of a strategy for information communication at the molecular level rather than to focus on practical applications, this system presents a number of interesting features, such as: (i) all outputs are encoded by optical signals (fluorescence); (ii) output reading by fluorescence spectroscopy does not affect the state of the logic gate; (iii) reconfigurability (currently limited to the solution phase) increases efficiency by reducing the number of logic devices required to form a multifunctional circuit, and (iv) operability (set/reset) at the solid state is the first step toward buildable data memory that can be achieved by integrating single "switchable" cells to form a complex circuit, where acid or base inputs write data along the array, and the output of an upstream gate provides the input for a downstream gate in a functional device.

\section{REFERENCES}

Andréasson, J., and Pischel, U. (2015). Molecules with a sense of logic: a progress report. Chem. Soc. Rev. 44, 1053-1069. doi: 10.1039/c4cs00342j

Baruah, M., Qin, W., Flors, C., Hofkens, J., Vallée, R. A. L., Beljonne, D., et al. (2006). Solvent and $\mathrm{pH}$ dependent fluorescent properties of a dimethylaminostyryl borondipyrromethene dye in solution. J. Phys. Chem. A 110, 5998-6009. doi: 10.1021/jp054878u

Baytekin, H. T., and Akkaya, E. U. (2000). A molecular NAND gate based on Watson-Crick base pairing. Org. Lett. 2, 1725-1727. doi: 10.1021/ol005873c

Coskun, A., Deniz, E., and Akkaya, E. U. (2005). Effective PET and ICT switching of boradiazaindacene emission: a unimolecular, emission-mode, molecular half-subtractor with reconfigurable logic gates. Org. Lett. 7, 5187-5189. doi: $10.1021 / \mathrm{ol} 052020 \mathrm{~h}$

Costero, A. M., Parra, M., Gil, S., and Gaviña, P. (2018). "BODIPY core as signaling unit in chemosensor design," in BODIPY Dyes - A Privilege Molecular Scaffold with Tunable Properties, eds. R. Bañuelos-Prieto, and J. Sola Llano (London: IntechOpen).

Daly, B., Ling, J., and de Silva, A. P. (2014). Information gathering and processing with fluorescent molecules. Front. Chem. Sci. Eng. 8, 240-251. doi: 10.1007/s11705-014-1432-z

de Silva, A. P. (2012a). Molecular and Supramolecular Information Processing. ed E. Katz. Weinheim: Wiley-VCH Verlag GmbH \& Co. KGaA.

de Silva, A. P. (2012b). Molecular Logic-based Computation. Cambridge: Royal Society of Chemistry.

de Silva, A. P., and Uchiyama, S. (2007). Molecular logic and computing. Nat. Nanotechnol. 2:399. doi: 10.1038/nnano.2007.188

de Silva, P. A., and McClenaghan, N. D. (2002). Simultaneously multiplyconfigurable or superposed molecular logic systems composed of ICT (internal charge transfer) chromophores and fluorophores integrated with

\section{DATA AVAILABILITY STATEMENT}

The datasets generated for this study are included in the article/Supplementary Material.

\section{AUTHOR CONTRIBUTIONS}

SI conceived the project and wrote the manuscript. SI, LT, and $\mathrm{GH}$ designed the experiments. LT performed the experiments and analysis. ND, GH, and SI supervised the project. All authors contributed to project discussions.

\section{FUNDING}

We thank the Natural Sciences and Engineering Council of Canada (Discovery Grant) and Ryerson University (start-up funds) for supporting our research program. This research has also been supported by the Ryerson University Faculty of Science Dean's Research Fund. ND acknowledges the award of a Ryerson Graduate Scholarship.

\section{SUPPLEMENTARY MATERIAL}

The Supplementary Material for this article can be found online at: https://www.frontiersin.org/articles/10.3389/fchem. 2020.00470/full\#supplementary-material

Supplementary Material: Absorption spectra; FTIR spectra; DR spectrum of a functionalized glass slide; Emission spectra of an unfunctionalized control slide; Synthetic schemes.

one- or two-ion receptors. Chem. A Eur. J. 8, 4935-4945. doi: 10.1002/15213765(20021104)8:21<4935::AID-CHEM4935>3.0.CO;2-2

Deschamps, J.-P., Valderrama, E., and Terés, L. (2017). Digital Systems. (Cham: Springer International Publishing).

Erbas-Cakmak, S., Kolemen, S., Sedgwick, A. C., Gunnlaugsson, T., James, T. D., Yoon, J., et al. (2018). Molecular logic gates: the past, present and future. Chem. Soc. Rev. 47, 2228-2248. doi: 10.1039/C7CS00491E

Feringa, B. L., and Browne, W. R. (eds.). (2011). Molecular Switches. Weinheim: Wiley-VCH Verlag GmbH \& Co. KGaA.

Ghatak, K. P., and Mitra, M. (2019). Nanomaterials, Volume 1: Electronic Properties. (Berlin: Walter de Gruyter GmbH).

Gibson, J. (2013). Electronic Logic Circuits. London: Routledge.

Hwang, E. (2016). Digital Logic and Microprocessor Design with Interfacing. Boston: Cengage Learning.

International Roadmap for Devices and Systems Executive Summary (2018). Available online at: https://irds.ieee.org/editions/2018

Jeong, Y., and Yoon, J. (2012). Recent progress on fluorescent chemosensors for metal ions. Inorganica Chim. Acta 381, 2-14. doi: 10.1016/j.ica.2011.09.011

Jiang, X. J., and Ng, D. K. P. (2014). Sequential logic operations with a molecular keypad lock with four inputs and dual fluorescence outputs. Angew. Chemie. Int. Ed. 53, 10481-10484. doi: 10.1002/anie.201406002

Kaur, P., and Singh, K. (2019). Recent advances in the application of BODIPY in bioimaging and chemosensing. J. Mater. Chem. C 7, 11361-11405. doi: 10.1039/c9tc03719e

Kulyk, B., Taboukhat, S., Akdas-Kilig, H., Fillaut, J. L., Karpierz, M., and Sahraoui, B. (2017). Tuning the nonlinear optical properties of BODIPYs by functionalization with dimethylaminostyryl substituents. Dye. Pigment. 137, 507-511. doi: 10.1016/j.dyepig.2016.10.045

Leen, V., and Dehaen, W. (2012). Fluorescent indicators based on BODIPY. Chem. Soc. Rev 41, 1130-1172. doi: 10.1039/C1CS15132K 
Lipiansky, E. (2012). Electrical, Electronics, and Digital Hardware Essentials for Scientists and Engineers. Hoboken, NJ: John Wiley \& Sons, Inc.

Margulies, D., Melman, G., Felder, C. E., Arad-Yellin, R., and Shanzer, A. (2004). Chemical input multiplicity facilitates arithmetical processing. J. Am. Chem. Soc. 126, 15400-15401. doi: 10.1021/ja0453329

Mathew, P. T., and Fang, F. (2018). Advances in molecular electronics: a brief review. Engineering 4, 760-771. doi: 10.1016/j.eng.2018.11.001

Reinsel, D., Gantz, J., and Rydning, J. (2018). The digitization of the world from edge to core - International Data Corporation (IDC). Available online at: https://www.seagate.com/files/www-content/our-story/trends/files/ idc-seagate-dataage-whitepaper.pdf

Rurack, K., Kollmannsberger, M., and Daub, J. (2001). Molecular switching in the near infrared (NIR) with a functionalized borondipyrromethene dye. Angew. Chemie Int. Ed. 40, 385-387. doi: 10.1002/ 1521-3773(20010119)40:2<385::AID-ANIE385>3.0.CO;2-F

Sarid, D., and Schechtman, B. H. (2007). A roadmap for optical data storage applications. Opt. Photonics News 18, 32-37. doi: 10.1364/OPN.18.5.000032

Shi, D. (2015). Nanomaterials and Devices. Oxford: Elsevier.

Shiraishi, Y., Tokitoh, Y., and Hirai, T. (2005). A fluorescent molecular logic gate with multiply-configurable dual outputs. Chem. Commun. 8, 5316-5318. doi: $10.1039 / \mathrm{b} 510800 \mathrm{~d}$

Stojanovic, M. N., Stefanovic, D., and Rudchenko, S. (2014). Exercises in molecular computing. Acc. Chem. Res. 47, 1845-1852. doi: 10.1021/ar5000538

Sun, L., Diaz-Fernandez, Y. A., Gschneidtner, T. A., Westerlund, F., LaraAvila, S., and Moth-Poulsen, K. (2014). Single-molecule electronics: from chemical design to functional devices. Chem. Soc. Rev. 43, 7378-7411. doi: $10.1039 / \mathrm{c} 4 \mathrm{cs} 00143 \mathrm{e}$

Swaminathan, H., and Balasurbamanian, K. (2019). Design of "turn-ON and turn-OFF” fluorescence switching based photonic logic gates through multiple input-output models by MoS2 quantum dots. J. Colloid Interface Sci. 540, 258-264. doi: 10.1016/j.jcis.2019.01.004

Szaciłowski, K. (2012). Infochemistry. Chichester: John Wiley \& Sons, Ltd.

Vollath, D. (2013). Nanomaterials: An Introduction to Synthesis, Properties and Applications, 2nd Edn. Weinheim: Wiley VCH.

Wagner, B. D., Arnold, A. E., Gallant, S. T., Grinton, C. R., Locke, J. K., Mills, N. D., et al. (2018). The polarity sensitivity factor of some fluorescent probe molecules used for studying supramolecular systems and other heterogeneous environments. Can. J. Chem. 96, 629-635. doi: 10.1139/cjc-2017-0727

Wu, Q., Zhang, T., Peng, Q., Wang, D., and Shuai, Z. (2014). Aggregation induced blue-shifted emission-the molecular picture from a QM/MM study. Phys. Chem. Chem. Phys. 16, 5545-5552. doi: 10.1039/c3cp54910k

Yu, Y. H., Descalzo, A. B., Shen, Z., Röhr, H., Liu, Q., Wang, Y. W., et al. (2006). Mono- and di(dimethylamino)styryl-substituted borondipyrromethene and borondiindomethene dyes with intense near-infrared fluorescence. Chem. - An Asian J. 1, 176-187. doi: 10.1002/asia.200600042

Zhang, B. (2018). Physical Fundamentals of Nanomaterials. Oxford: Elsevier.

Conflict of Interest: The authors declare that the research was conducted in the absence of any commercial or financial relationships that could be construed as a potential conflict of interest.

Copyright (c) 2020 Trifoi, Hodgson, Dogantzis and Impellizzeri. This is an openaccess article distributed under the terms of the Creative Commons Attribution License (CC BY). The use, distribution or reproduction in other forums is permitted, provided the original author(s) and the copyright owner(s) are credited and that the original publication in this journal is cited, in accordance with accepted academic practice. No use, distribution or reproduction is permitted which does not comply with these terms. 